\title{
Steady motions of nonhomogeneous ellipsoid on horizontal plane
}

\author{
Munitsyna M.A. \\ Moscow Institute of Physics and Technology \\ MIPT \\ Dolgoprudny, Russia \\ munitsyna@gmail.com
}

\author{
Munitsyn A.I. \\ Moscow Power Engineering Institute \\ MPEI \\ Moscow, Russia \\ munitsyn@rambler.ru
}

\begin{abstract}
The dynamics of a heavy nonhomogeneous ellipsoid of rotation on a horizontal fixed plane with friction is analysed. It is assumed that the mass centre of the spheroid coincides with its geometric center and that the geometric axis of symmetry is the axis of dynamic symmetry. The frictional force is proportional to the velocity of the point of contact with the plane; the frictional moment is equal to zero. The steady motions of the ellipsoid are investigated. The conditions of stability are obtained. The results are compared with the corresponding results in the case of an absolutely smooth and absolutely rough surface. The unsteady motions of the spheroid are investigated numerically. The similar results for the special case of homogeneous ellipsoid have been given earlier [1].
\end{abstract}

Keywords - stability; steady motions; viscous friction

\section{STATEMENT OF THE PROBLEM}

The problem of the undetached motion of a homogeneous heavy body, having the form of a spheroid on a horizontal plane 6 is considered. It is assumed that at the point of contact of the body with the plane, in addition to the normal reaction, there is also a force proportional to the velocity of this point of the body and acting in the opposite direction (the viscous friction force).

In a moving system of coordinates $C \xi_{1} \xi_{2} \xi_{3}$ with origin at the centre of mass of the spheroid and with the axes directed along its principal central axes of inertia, the equations of motion of the body have the form:

$$
\begin{aligned}
& m \dot{\mathbf{v}}+[\boldsymbol{\omega}, m \mathbf{v}]=-m g \boldsymbol{\gamma}+N \boldsymbol{\gamma}+\mathbf{F} \\
& J \dot{\boldsymbol{\omega}}+[\boldsymbol{\omega}, J \boldsymbol{\omega}]=[\mathbf{r}, N \boldsymbol{\gamma}+\mathbf{F}] \\
& \dot{\boldsymbol{\gamma}}+[\boldsymbol{\omega}, \boldsymbol{\gamma}]=0 \\
& (\mathbf{u}, \boldsymbol{\gamma})=0
\end{aligned}
$$

Equations (1) and (2) are the theorems of the change in the momentum and in the angular momentum. Equation (3) is the constancy of the unit vector of the ascending vertical $\gamma$; equation (3) is the condition of non-detachment of the motion of the body. Here $m$ is the mass of the spheroid; $\mathbf{v}$ is the velocity vector of its centre of the mass of the spheroid; $\boldsymbol{\omega}$ is the angular velocity vector; $g$ is the gravitational acceleration; $N$ is the value of the normal component of the reaction of the supporting plane; $J=\operatorname{diag}(A, A, C)$ is the central inertia tensor of the spheroid; $\mathbf{r}$ is the radius vector of the velocity of the point of contact of the spheroid with the plane; $\mathbf{u}=\mathbf{v}+[\boldsymbol{\omega}, \mathbf{r}]$ is the velocity of this point; $F=-m \kappa u$ is the viscous friction force, and $\kappa$ is the friction coefficient.

Value $\kappa=0$ corresponds to the case of an absolutely smooth surface. Moreover, in the limit case when $\kappa=\rightarrow \infty$ the viscous friction force produces non-holonomic coupling, corresponding to the case of an absolutely rough surface [2], [3].

The equation of the surface of a spheroid with equatorial radius $a$ and axial radius $c$ has the form:

$$
f(\mathbf{r})=\left(\xi_{1}^{2}+\xi_{2}^{2}\right) / a^{2}+\xi_{3}^{2} / c^{2}=0
$$

and the following equality holds:

$$
\gamma=-\frac{\operatorname{gradf}(\mathbf{r})}{|\operatorname{gradf}(\mathbf{r})|}
$$

An expression for the normal reaction of the plane can be obtained from Eq. (1) using relation (4):

$$
\begin{gathered}
N=m(g+h(\mathbf{r}, \boldsymbol{\omega})) \\
h(\mathbf{r}, \boldsymbol{\omega})=([\mathbf{r}, \dot{\boldsymbol{\omega}}]+[\dot{\mathbf{r}}, \boldsymbol{\omega}], \boldsymbol{\gamma})+([\boldsymbol{\omega}, \mathbf{r}],[\boldsymbol{\omega}, \boldsymbol{\gamma}])
\end{gathered}
$$

and the equations of motion of the spheroid take the form:

$$
\begin{gathered}
\dot{\mathbf{u}}+[\boldsymbol{\omega}, \mathbf{u}]=-[\dot{\mathbf{r}}, \boldsymbol{\omega}]-[\mathbf{r}, \dot{\boldsymbol{\omega}}]+[\boldsymbol{\omega},[\boldsymbol{\omega}, \mathbf{r}]]+h(\mathbf{r}, \boldsymbol{\omega}) \boldsymbol{\gamma}+\mathbf{F} \\
J \boldsymbol{\omega}+[\boldsymbol{\omega}, J \boldsymbol{\omega}]=(g+h(\mathbf{r}, \boldsymbol{\omega}))[\mathbf{r}, \boldsymbol{\gamma}]+[\mathbf{r}, \mathbf{F}]+\mathbf{M} \\
\dot{\boldsymbol{\gamma}}+[\boldsymbol{\omega}, \boldsymbol{\gamma}]=0
\end{gathered}
$$

Taking relation (5) into account, the system of equations (6) is a closed system of differential equations in $\mathbf{u}, \boldsymbol{\omega}$ and $\gamma$; it has a geometrical integral $\gamma^{2}=1$ and particular integral (4).

The resulting system has the following solutions:

$$
\begin{gathered}
\gamma_{1}=\gamma_{2}=0, \quad \gamma_{3}= \pm 1 \\
\omega_{1}=\omega_{2}=0, \quad \omega_{3}=\omega=\text { const }
\end{gathered}
$$

$$
\begin{gathered}
\gamma_{1}=\sin \psi, \gamma_{2}=\cos \psi, \psi=\text { const }, \quad \gamma_{3}=0 \\
\omega_{1}=\omega \gamma_{1}, \omega_{2}=\omega \gamma_{2}, \omega_{3}=0 \\
\gamma_{1}=\sin \theta \sin \omega t, \gamma_{2}=\sin \theta \cos \omega t, \gamma_{3}=0 \\
\omega_{1}=\omega_{2}=0, \quad \omega_{3}=\omega=\text { const }
\end{gathered}
$$




$$
\begin{gathered}
\gamma_{1}=\sin \theta \sin \omega_{0} t, \gamma_{2}=\sin \theta \cos \omega_{0} t \\
\gamma_{3}=\cos \theta=\text { const } \\
\omega_{1}=\omega \gamma_{1}, \omega_{2}=\omega \gamma_{2}, \omega_{3}=\omega_{0}+\omega \gamma_{3}
\end{gathered}
$$

There is no slippage $(\mathbf{u}=0)$ in these solutions, and the value of the normal reaction is equal to the body weight ( $N=m g$ ).

Solutions (7) correspond to uniform rotations of the ellipsoid around the axis of dynamic symmetry, coinciding with the vertical. Solutions (8) correspond to uniform rotations of the ellipsoid around the equatorial radius, coinciding with the vertical, and solutions (9) correspond to uniform rolling of the ellipsoid with an equatorial cross section along the straight line. Solutions (10) correspond to regular precessions of the ellipsoid, in which its centre of mass is fixed, while its point of contact with the support plane describes a circle with angular velocity $\omega$ on the plane and a circle with angular velocity $\omega_{0}$ on the surface of the ellipsoid [4].

\section{THE STABILITY OF THE SOLUTIONS}

Further, the stability of solutions (7) is investigated. The linearized equations of the perturbed motion in the neighbourhood of the solutions have the form:

$$
\begin{aligned}
& \delta \dot{u}_{1} \pm c \delta \dot{\omega}_{2}-R_{c} \omega \delta \dot{\gamma}_{2}+ \\
& +\kappa \delta u_{1}-\omega \delta u_{2} \pm c \omega \delta \omega_{1}-R_{c} \omega^{2} \delta \gamma_{1}=0 \\
& \delta \dot{u}_{2} \mp c \delta \dot{\omega}_{1}+R_{c} \omega \delta \dot{\gamma}_{1}+ \\
& +\omega \delta u_{1}+\kappa \delta u_{2} \pm c \omega \delta \omega_{2}-R_{c} \omega^{2} \delta \gamma_{2}=0 \\
& \delta \dot{u}_{3}+\kappa \delta u_{3}=0 \\
& A \delta \dot{\omega}_{1} \pm m c \kappa \delta u_{2}+\omega(C-A) \omega_{2} \pm m g\left(R_{c}-c\right) \delta \gamma_{2}=0 \\
& A \delta \dot{\omega}_{2} \mp m c \kappa \delta u_{1}-\omega(C-A) \omega_{1} \mp m g\left(R_{c}-c\right) \delta \gamma_{1}=0 \\
& \delta \dot{\omega}_{3}=0 \\
& \delta \dot{\gamma}_{1} \pm \omega_{2}-\omega \delta \gamma_{2}=0 \\
& \delta \dot{\gamma}_{2} \mp \omega_{1}+\omega \delta \gamma_{1}=0 \\
& \delta \dot{\gamma}_{3}=0
\end{aligned}
$$

In the new variables:

$$
U=\delta u_{1}+i \delta u_{2}, \quad \Omega=\delta \omega_{1}+i \delta \omega_{2}, \quad \gamma=\delta \gamma_{1}+i \delta \gamma_{2}
$$

these equations take the form:

$$
\begin{gathered}
\dot{U} \pm c i \dot{\Omega}-R_{c} \omega i \dot{\Gamma}+(\kappa-\omega i) U \pm c \omega \Omega-R_{c} \omega^{2} \Gamma=0 \\
A \dot{\Omega} \pm c \kappa U+(C-A) \omega i \Omega \pm\left(R_{c}-c\right) g i \Gamma=0 \\
\dot{\Gamma} \pm i \Omega \pm \omega i \Gamma=0 \\
\delta \dot{u}_{3}=0, \quad \delta \dot{\omega}_{3}=0, \quad \delta \dot{\gamma}_{3}=0
\end{gathered}
$$

Here $R_{c}=a^{2} / c$ is the radius of curvature of the spheroid surface at the point of contact with plane $\delta u_{k}, \delta \omega_{k}$ and $\delta \gamma_{k}$ ( $k \in\{1,2,3\}$ ) are perturbations of the corresponding variables.

Coefficients of the characteristic equation $\chi(\lambda)=0$ are

$$
\chi(\lambda)=a_{0} \lambda^{3}+\left(a_{1} \kappa+i b_{1}\right) \lambda^{2}+\left(a_{2}+i b_{2} \kappa\right) \lambda+\left(a_{3} \kappa+i b_{3}\right)
$$

obtained from the first three equations of the system have the form:

$$
\begin{aligned}
& a_{0}=A>0 \\
& a_{1}=\left(A+m c^{2}\right) \\
& b_{1}=(3 A-C) \omega \\
& a_{2}=(2 C-3 A) \omega^{2}+m g(R-c) \\
& b_{2}=(2 A-C-m c(R-2 c)) \omega \\
& a_{3}=\left(\left(C-A \omega^{2}+m(R-c)\left(c \omega^{2}+g\right)\right)\right. \\
& b_{3}=\left(m g(R-c)+(C-A) \omega^{2}\right) \omega
\end{aligned}
$$

The limit of the region of stability in the plane of the parameters $(c / a, \omega)$ is given by the equation $\chi(\xi i)=0$, Which is equivalent to the system:

$$
\begin{aligned}
& a_{0} \xi^{3}+b_{1} \xi^{2}-a_{2} \xi-b_{3}=0 \\
& a_{1} \xi^{2}+b_{2} \xi-a_{3}=0
\end{aligned}
$$

with the solution:

$$
\begin{gathered}
\xi=\frac{a_{0} a_{3} b_{2}+a_{1}^{2} b_{3}-a_{1} a_{3} b_{1}}{a_{0} a_{1} a_{3}+a_{0} b_{2}^{2}-a_{1}^{2} a_{2}-a_{1} b_{1} b_{2}} \\
\omega^{2}=\omega_{(1)}^{2}=\frac{m g c^{3}\left(c^{2}-a^{2}\right)}{a^{2}\left(C c^{2}-A a^{2}\right)}
\end{gathered}
$$

Moreover, when $\omega=0$, the considered solutions are stable for oblate spheroids $(a<c)$.

Hence when the following condition is satisfied

$$
(c-a)\left(\omega^{2}-\omega_{(1)}^{2}\right)>0,
$$

solutions (7) are asymptotically stable with respect to variables $u_{k}, \omega_{k}$, and $\gamma_{k}(k \in\{1,2\})$. Moreover, asymptotic stability with respect to variables $u_{3}$ and $\gamma_{3}$ occurs (the constants of the integrals are not perturbed) and non-asymptotic stability with respect to variable $\omega_{3}$.

To investigate the stability of rotations of the form (8), without loss of generality, one can put $\gamma_{1}=1, \gamma_{2}=0$. The linearized equations of the perturbed motion then have the form

$$
\begin{aligned}
& \delta \dot{u}_{1}+\kappa \delta u_{1}=0 \\
& \delta \dot{u}_{2}+a \delta \dot{\omega}_{3}-R_{a} \omega \delta \dot{\gamma}_{3}+\kappa \delta u_{2}-\omega \delta u_{3}+a \omega \delta \omega_{2}-a \omega^{2} \delta \gamma_{2}=0 \\
& \delta \dot{u}_{3}-a \delta \dot{\omega}_{2}+a \omega \delta \dot{\gamma}_{2}+\omega \delta u_{2}+\kappa \delta u_{3}+a \omega \delta \omega_{3}-R_{a} \omega^{2} \delta \gamma_{3}=0 \\
& \delta \dot{\omega}_{1}=0 \\
& A \delta \dot{\omega}_{2}+m a \kappa \delta u_{2}-(C-A) \omega \delta \omega_{3}+m g\left(R_{a}-a\right) \delta \gamma_{3}=0 \\
& C \delta \dot{\omega}_{3}-m a \kappa \delta u_{2}=0 \\
& \delta \dot{\gamma}_{1}=0 \\
& \delta \dot{\gamma}_{2}+\delta \omega_{3}-\omega \delta \gamma_{3}=0 \\
& \delta \dot{\gamma}_{3}-\delta \omega_{2}+\omega \delta \gamma_{2}=0
\end{aligned}
$$

where $R_{a}=c^{2} / a$ is the radius of curvature of the spheroid surface at the point of contact with the plane.

The characteristic equation

$$
\begin{gathered}
\lambda^{3}(\lambda+\kappa) P_{5}(\lambda)=0 \\
P_{5}(\lambda)=a_{01} \lambda^{5}+a_{11} \lambda^{4}+a_{21} \lambda^{3}+a_{30} \lambda^{2}+a_{41} \lambda+a_{51}
\end{gathered}
$$




$$
\begin{aligned}
a_{01}= & A C>0 \\
a_{11}= & \left(m a^{2}(A+C)+2 A C\right) \kappa>0 \\
a_{21}= & \left(m a^{2}\left(m a^{2}+A+C\right)+A C\right) \kappa^{2}+ \\
& +2 A C \omega^{2}+m g C\left(R_{a}-a\right) \\
a_{31}= & \left(\left(2 A\left(m a^{2}+C\right)-m C\left(R_{a}-a\right)\right) \omega^{2}+\right. \\
& \left.+m g\left(R_{a}-a\right)\left(m a^{2}+2 C\right)\right) \kappa \\
a_{41}= & \left(a^{2}\left(m a^{2}+A+C\right)+A C\right) \omega^{2} \kappa^{2}+ \\
& +m g\left(R_{a}-a\right)\left(m a^{2}+C\right) \kappa^{2} \\
& +C \omega^{2}\left(A \omega^{2}+m g\left(R_{a}-a\right)\right) \\
a_{51}= & m\left(a^{2}\left(A a-C R_{a}\right) \omega^{2}+m g a^{2}\left(R_{a}-a\right)\right) \omega^{2} \kappa
\end{aligned}
$$

has three zero roots. The real parts of all the remaining roots are negative when the following conditions are satisfied $[4,5]$ :

$$
\begin{gathered}
a_{31}>0, \quad a_{51}>0, \quad a_{11} a_{21}-a_{01} a_{31}>0 \\
\left(a_{11} a_{21}-a_{01} a_{51}\right)\left(a_{31} a_{41}-a_{21} a_{51}\right)-\left(a_{01} a_{51}-a_{11} a_{41}\right)^{2}>0
\end{gathered}
$$

The regions of stability in the plane of parameters $(\omega, \kappa)$, corresponding to conditions (12) in the case of homogeneous ellipsoid have been given earlier [1]. For nonhomogeneous case, let us assume that the small value of friction coefficient confines itself to considering in (12) in terms of the first order of smallness.

Hence, conditions (12) have the form:

$$
\begin{gathered}
\left((1-\sigma)\left(v_{2}-1\right) v_{1}^{2}+\sigma\left(v_{1}^{2}+v_{2}\right)\right) \mu+2 v_{2}\left(v_{2}+\sigma-1\right)>0 \\
1-\sigma) /\left(v_{2}-1\right)>0 \\
\left(2+\mu v_{1}^{2}\right) \sigma+\left(v_{2}+\sigma-1\right) \mu>0 \\
v_{1}^{2} \sigma+v_{2}+\sigma-1>0
\end{gathered}
$$

and are equivalent to the inequality:

$$
(\sigma-1) /\left(v_{2}-1\right)<0 .
$$

In this case, the following parameters are introduced:

$$
\begin{gathered}
\mu=\frac{m a^{2}}{A}, \quad \sigma=\frac{\omega^{2}}{\omega_{(2)}^{2}}, \quad v_{1}=\frac{c}{a}, \quad v_{2}=\frac{C c^{2}}{A a^{2}} \\
\omega_{(2)}^{2}=\frac{m g a\left(c^{2}-a^{2}\right)}{C c^{2}-A a^{2}}
\end{gathered}
$$

$\sigma$ is the dimensionless angular velocity; $v_{1}$ is the parameter characterizing the degree of protateness or oblateness of the ellipsoid; $v_{2}$ is the dimensionless parameter characterizing the mass distribution in the ellipsoid.

In the case of an absolutely smooth surface $(\kappa=0)$, equation (11) takes the form:

$$
\lambda^{5}\left(\lambda^{2}+\omega^{2}\right)\left(A a\left(\lambda^{2}+\omega^{2}\right)+m g\left(c^{2}-a^{2}\right)\right)=0,
$$

and rotations (8) are stable in the first approximation for prolate ellipsoid ( $c>a)$ and for oblate ellipsoid $(c<a)$ when the following condition is satisfied:

$$
\omega^{2}>\frac{m g\left(a^{2}-c^{2}\right)}{A a} .
$$

In the case of an absolutely rough surface $(\kappa=\rightarrow \infty)$, the number of degrees of freedom is reduced, and (11) takes the form:

$$
\lambda^{4}\left(a\left(A+m a^{2}\right)\left(\lambda^{2}+\omega^{2}\right)+m g\left(c^{2}-a^{2}\right)\right)=0
$$

The corresponding stability conditions

$$
a\left(A+m a^{2}\right) \omega^{2}+m g\left(c^{2}-a^{2}\right)>0
$$

are identical with those obtained earlier [6].

Further, the stability of solutions (9) is investigated. The linearized equations of the perturbed motion in the neighbourhood of the solutions have the form:

$$
\begin{aligned}
& \delta \dot{u}_{1}-a \gamma_{2} \delta \dot{\omega}_{3}+\kappa \delta u_{1}-\omega \delta u_{2}=0 \\
& \delta \dot{u}_{2}+a \gamma_{1} \delta \dot{\omega}_{3}+\omega \delta u_{1}+\kappa \delta u_{2}=0 \\
& \delta \dot{u}_{3}+a \gamma_{2} \delta \dot{\omega}_{1}-a \gamma_{1} \delta \dot{\omega}_{2}+\kappa \delta u_{3}=0 \\
& A \delta \dot{\omega}_{1}-m a \gamma_{2} \kappa \delta u_{3}+(C-A) \omega \delta \omega_{2}+\gamma_{2} m g(R-a) \delta \gamma_{3}=0 \\
& A \delta \dot{\omega}_{2}+m a \gamma_{1} \kappa \delta u_{3}-(C-A) \omega \delta \omega_{1}-\gamma_{1} m g(R-a) \delta \gamma_{3}=0 \\
& C \delta \dot{\omega}_{3}+m a\left(\gamma_{2} \delta u_{1}-\gamma_{1} \delta u_{2}\right) \kappa=0 \\
& \delta \dot{\gamma}_{1}-\gamma_{2} \delta \omega_{3}-\omega \delta \gamma_{1}=0 \\
& \delta \dot{\gamma}_{2}+\gamma_{1} \delta \omega_{3}+\omega \delta \gamma_{2}=0 \\
& \delta \dot{\gamma}_{3}+\gamma_{2} \delta \omega_{1}-\gamma_{1} \delta \omega_{2}=0 \\
& \left(\gamma_{1}=\sin \omega t, \gamma_{2}=\cos \omega t\right)
\end{aligned}
$$

In the new variables:

$$
\begin{array}{cc}
U_{1}=\gamma_{1} \delta u_{1}+\gamma_{2} \delta u_{2}, & U_{2}=\gamma_{2} \delta u_{1}-\gamma_{1} \delta u_{2} \\
\Omega_{1}=\gamma_{1} \delta \omega_{1}+\gamma_{2} \delta \omega_{2}, & \Omega_{2}=\gamma_{2} \delta \omega_{1}-\gamma_{1} \delta \omega_{2} \\
\Gamma_{1}=\gamma_{1} \delta \gamma_{1}+\gamma_{2} \delta \gamma_{2}, & \Gamma_{2}=\gamma_{2} \delta \gamma_{1}-\gamma_{1} \delta \gamma_{2} \\
\Gamma_{3}=\delta \gamma_{3}, \quad \Omega_{3}=\delta \omega_{3}, \quad U_{3}=\delta u_{3}
\end{array}
$$

this equations take the form

$$
\begin{aligned}
& \dot{U}_{1}+\kappa U_{1}=0 \\
& C \dot{U}_{2}+\left(C+m a^{2}\right) \kappa U_{2}=0 \\
& A \dot{U}_{3}+\left(A+m a^{2}\right) \kappa U_{3}-a(C-A) \omega \Omega_{1}-m g( \\
& \dot{\Omega}_{1}-C \omega \Omega_{2} / A=0 \\
& A \dot{\Omega}_{2}-m a \kappa U_{3}+C \omega \Omega_{1}+m g(R-a) \Gamma_{3}=0 \\
& \dot{\Omega}_{3}+m a \kappa U_{2} / C=0 \\
& \dot{\Gamma}_{1}=0 \\
& \dot{\Gamma}_{2}-\Omega_{3}=0 \\
& \dot{\Gamma}_{3}+\Omega_{2}=0 \\
& \text { The characteristic equation of this system } \\
& \lambda^{4}(\lambda+\kappa)\left(C \lambda+\left(C+m a^{2}\right) \kappa\right) P_{3}(\lambda)=0 \\
& P_{3}(\lambda)=\left(a_{02} \lambda^{3}+a_{12} \lambda^{2}+a_{22} \lambda+a_{32}\right) \\
& a_{02}=A^{2}>0 \\
& a_{12}=A\left(A+m a^{2}\right) \kappa>0 \\
& a_{22}=C^{2} \omega^{2}+m g A\left(R_{a}-a\right) \\
& a_{32}=\left(C\left(C+m a^{2}\right) \omega^{2}+m g A\left(R_{a}-a\right)\right) \kappa
\end{aligned}
$$$$
A \dot{U}_{3}+\left(A+m a^{2}\right) \kappa U_{3}-a(C-A) \omega \Omega_{1}-m g(R-a) \Gamma_{3}=0
$$

has four zero roots. The real parts of all the remaining roots are negative when conditions $a_{32}>0$ and inequality

$$
a_{12} a_{22}-a_{02} a_{32}=C(C-A) \omega^{2}+m g A\left(R_{a}-a\right)>0
$$

are satisfied. They are always fulfilled for an ellipsoid with parameters $c>a, C>A$; never satisfied for an ellipsoid with parameters $c<a, C<A$; and have the form 


$$
\begin{aligned}
& (c-a)\left(\omega^{2}-\omega_{(3)}^{2}\right)<0 \\
& \omega_{(3)}^{2}=\frac{m g\left(c^{2}-a^{2}\right)}{(A-C) a^{2}} \frac{A}{C}
\end{aligned}
$$

for an ellipsoid which parameters satisfy the condition:

$$
(c-a)(C-A)<0 \text {. }
$$

In the case of an absolutely smooth surface $(\kappa=0)$, equation (14) is reduced to the form:

$$
\lambda^{7}\left(a A^{2} \lambda^{2}+a C^{2} \omega^{2}+m g A\left(c^{2}-a^{2}\right)\right)=0
$$

and solution (9) is stable in the first approximation for prolate ellipsoid $(c>a)$ and for oblate ellipsoid $(c<a)$ when the following condition is satisfied:

$$
\omega^{2}>\frac{m g A\left(a^{2}-c^{2}\right)}{C^{2} a}
$$

In the case of an absolutely rough surface $(\kappa=\rightarrow \infty)$, equation (14) is reduced to the form:

$\lambda^{4}\left(a A\left(A+m a^{2}\right) \lambda^{2}+a C\left(C+m a^{2}\right) \omega^{2}+m g\left(c^{2}-a^{2}\right) A\right)=0$,

and the corresponding stability conditions

$$
c C\left(C+m a^{2}\right) \omega^{2}+m g A\left(c^{2}-a^{2}\right)>0
$$

coincide with those obtained earlier [7].

Uniform rolling of an ellipsoid are stable in a first approximation for prolate ellipsoid $(c>a)$ and for oblate ellipsoid $(c<a)$ when the following condition is satisfied

$$
\omega^{2}>\frac{m g A\left(a^{2}-c^{2}\right)}{a C\left(C+m a^{2}\right)}
$$

Now, the stability of regular precessions of an ellipsoid around the center of mass (10) is investigated. In the variables:

$$
\begin{aligned}
& U_{1}=\gamma_{1} \delta u_{1}+\gamma_{2} \delta u_{2}+\gamma_{3} \delta u_{3} \\
& U_{2}=\gamma_{2} \delta u_{1}-\gamma_{1} \delta u_{2} \\
& U_{3}=\delta u_{3} \\
& \Omega_{1}=\gamma_{1} \delta \omega_{1}+\gamma_{2} \delta \omega_{2} \\
& \Omega_{2}=\gamma_{2} \delta \omega_{1}-\gamma_{1} \delta \omega_{2}-\omega \gamma_{2} \delta \gamma_{1}+\omega \gamma_{1} \delta \gamma_{2} \\
& \Omega_{3}=\delta \omega_{3} \\
& \Gamma_{1}=\gamma_{1} \delta \gamma_{1}+\gamma_{2} \delta \gamma_{2}+\gamma_{3} \delta \gamma_{3} \\
& \Gamma_{2}=\gamma_{2} \delta \gamma_{1}-\gamma_{1} \delta \gamma_{2} \\
& \Gamma_{3}=\delta \gamma_{3}-\gamma_{3} \Gamma_{1}
\end{aligned}
$$

the linearized equations of the perturbed motion in the neighbourhood of the solutions have the form

$$
\begin{aligned}
& \dot{U}_{1}+\kappa U_{1}=0 \\
& \dot{U}_{2}+c^{2} \rho^{-1} \cos \theta \dot{\Omega}_{1}-a^{2} \rho^{-1} \sin ^{2} \theta \dot{\Omega}_{3}+c^{2} \rho^{-1} \omega \dot{\Gamma}_{3}- \\
& -\omega \cos \theta U_{1}+\kappa U_{2}+\omega U_{3}+\omega \rho \Omega_{2}=0 \\
& \dot{U}_{3}+\rho \dot{\Omega}_{2}-\omega U_{2}+\kappa U_{3}-c^{2} \rho^{-1} \omega \cos \theta \Omega_{1}+ \\
& +a^{2} \rho^{-1} \omega \sin ^{2} \theta \Omega_{3}-c^{2} \rho^{-1} \omega^{2} \Gamma_{3}=0
\end{aligned}
$$

$A \dot{\Omega}_{1}-\kappa m c^{2} \rho^{-1} \cos \theta U_{2}+\left(A-C c^{2} a^{-2}\right) \omega \cos \theta \Omega_{2}=0$

$(A+B) \dot{\Omega}_{2}+A \omega \dot{\Gamma}_{2}+\kappa m c^{2} \rho^{-1} \cos \theta U_{1}-\kappa m \rho U_{3}-$

$-\left(A-C c^{2} a^{-2}\right) \omega \cos \theta \Omega_{1}+(C-A) \omega \sin ^{2} \theta \Omega_{3}-$

$-m g\left(c^{2}-a^{2}\right) \rho^{-1} \sin ^{2} \theta \cos \theta \Gamma_{1}-$

$-m g\left(c^{2}-a^{2}\right) \rho^{-1}\left(c^{2} \cos ^{4} \theta-a^{2} \sin ^{4} \theta\right) \rho^{-2} \Gamma_{3}=0$

$C \dot{\Omega}_{3}+\kappa m a^{2} \rho^{-1} U_{2}=0$

$\dot{\Gamma}_{1}=0$

$\dot{\Gamma}_{2}+\cos \theta \Omega_{1}-\sin ^{2} \theta \Omega_{3}+\omega \Gamma_{3}=0$

$\dot{\Gamma}_{3}+\Omega_{2}=0$

$$
B=m \frac{\left(c^{2}-a^{2}\right)^{2}}{4 \rho^{2}} \sin ^{2} 2 \theta
$$

The nonzero roots of the equation:

$$
\lambda^{4}\left(a_{03} \lambda^{5}+a_{13} \lambda^{4}+a_{23} \lambda^{3}+a_{33} \lambda^{2}+a_{43} \lambda+a_{53}\right)=0(17)
$$

have no positive real parts under conditions:

$$
\begin{gathered}
a_{13}>0, \quad a_{33}>0, \quad a_{53}>0 \\
a_{13} a_{23}-a_{03} a_{33}>0 \\
\left(a_{13} a_{23}-a_{03} a_{53}\right)\left(a_{33} a_{43}-a_{23} a_{53}\right)- \\
-\left(a_{03} a_{53}-a_{13} a_{43}\right)^{2}>0
\end{gathered}
$$

The regions of stability in the plane of the parameters $\left(\cos \theta_{0}, \kappa\right)$, corresponding to conditions (18) in the case of homogeneous ellipsoid, have been given earlier [8]. For the nonhomogeneous case, let us confine ourselves to the case of small friction, close to the sphere of the ellipsoid shape, and close to the spherical tensor of its inertia, i.e. the following quantities are assumed to be small:

$$
\begin{aligned}
& \varepsilon=\frac{\kappa^{2}\left(A+m a^{2}\right)^{2}}{4 m g A a}<1 \\
& \varepsilon_{1}=\frac{c}{a}-1<<1, \quad \varepsilon_{2}=\frac{C c^{2}}{A a^{2}}-1<<1
\end{aligned}
$$

Then conditions (18) can be written in the form:

$$
\begin{aligned}
& 2 \kappa A^{3}(\mu+1)+O\left(\varepsilon_{1}, \varepsilon_{2}\right)>0 \\
& 4 \kappa \mu g \frac{A^{3}(\mu+1)}{a} \cdot \frac{\varepsilon_{1}+O_{2}\left(\varepsilon_{1}, \varepsilon_{2}\right)}{\varepsilon_{2}}>0 \\
& 8 \kappa \mu^{3} g^{2} \gamma_{3}^{2}\left(1-\gamma_{3}^{2}\right) \frac{A^{3}}{a^{2}} \cdot \frac{\varepsilon_{1}^{2}\left(\varepsilon_{1}+2 \varepsilon_{2}\right)+O_{4}\left(\varepsilon_{1}, \varepsilon_{2}\right)}{\varepsilon_{2}}>0 \\
& 4 \kappa \mu g \frac{A^{6}(\mu+1)}{a} \cdot \frac{\varepsilon_{1}+O_{2}\left(\varepsilon_{1}, \varepsilon_{2}, \varepsilon\right)}{\varepsilon_{2}}>0 \\
& 64 \kappa^{2} \mu^{5} g^{4} \frac{A^{12}}{a^{2}} \cdot \frac{\varepsilon_{1}^{2}}{\varepsilon_{2}^{2}}\left(\left(3 \gamma_{3}^{2}-1\right)^{2} \varepsilon_{1}^{2}+\varepsilon O_{2}\left(\varepsilon_{1}, \varepsilon_{2}, \varepsilon\right)\right)>0
\end{aligned}
$$


In the case when the parameters of the ellipsoid satisfy condition (15), values $\varepsilon_{1}$ and $\varepsilon_{2}$ have one sign, conditions are satisfied everywhere except for a small neighborhood of point $\gamma_{3}^{2}=1 / 3$. As an example, in Fig. 1, there is the region of solution stability for the following parameters of the ellipsoid:

$$
\varepsilon_{1}=0.1, \quad \varepsilon_{2}=-0.1
$$

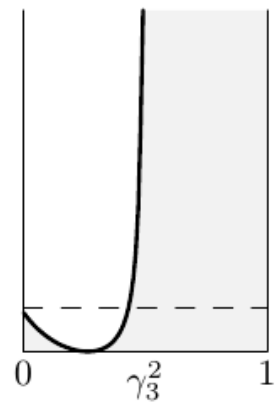

Fig. 1. Region of solution stability for the following parameters of the ellipsoid.

\section{NUMERICAL EXPERIMENTS}

Let us note that there are the unsteady motions in which, during transitens, the ellipsoid detaches from the plane. In such cases, Eqs. (1)-(4) describe the motion of the ellipsoid up to the instant when it detaches away from the plane, and Eqs. (1)-(3) $(\mathrm{N}=0)$ describe the free motion of the ellipsoid after it detaches and until the next instant when it comes to contact with the plane. Eqs. (1)-(3) are insufficient to determine the condition for constraint to occur, and they must be supplemented by the equation:

$$
\dot{\varepsilon}=(\mathbf{u}, \gamma)
$$

where $\varepsilon$ is the height above the plane of the lowest point of the ellipsoid, given by Eq. (5).

For the slightly deformed plane, Eq. (19) also holds in the case when the ellipsoid is in contact with the plane. Then, to determine the normal reaction, the Hertz' model is used [8]:

$$
N=\left\{\begin{array}{l}
\alpha|\varepsilon|^{3 / 2}, \quad \varepsilon<0 \\
0, \quad \varepsilon>0
\end{array}\right.
$$

where $\alpha$ is a constant, determined by the properties of the materials of the ellipsoid and the plane. The friction force is given by the equality:

$$
F=\left\{\begin{array}{l}
-m \kappa u, \quad \varepsilon<0 \\
0, \quad \varepsilon>0
\end{array}\right.
$$

The system of differential equations (1)-(3) and (19), taking relations (20) and (21) into account, is closed and completely describes the motion of the ellipsoid. Let us note that all the equations of this system have a Cauchy form, which simplifies the numerical integration algorithm of the Runge-Kutta method [9].
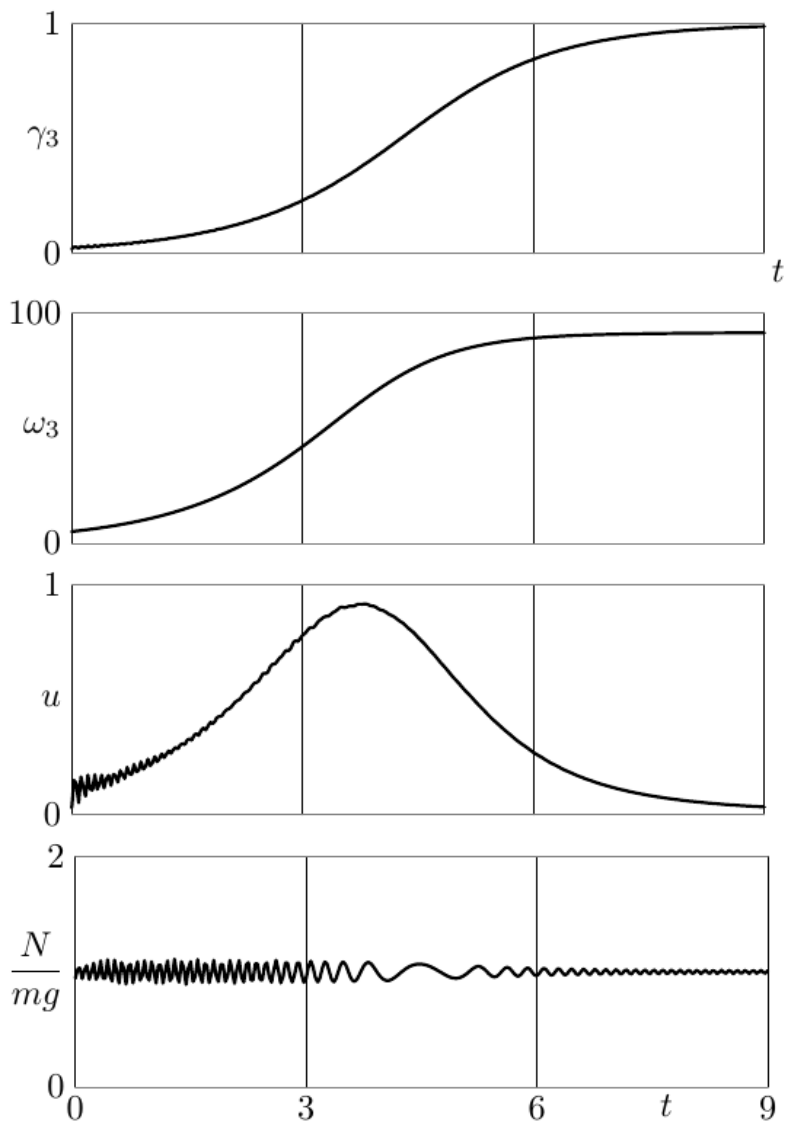

Fig. 2. Time dependences of the cosine of the nutation angle of the ellipsoid

Numerical experiments were carried out for the spheroid with the following parameters:

$m=2 \mathrm{~kg}, a=0.05 \mathrm{~m}, c=0.1 \mathrm{~m}$

$A=0.006 \mathrm{~kg} \cdot \mathrm{m}^{2}, C=0.001 \mathrm{~kg} \cdot \mathrm{m}^{2}$

on the plane with viscous friction coefficient $\kappa=3 s^{-1}$ and $\alpha=10^{5} \mathrm{~kg} /\left(\mathrm{s}^{2} \mathrm{~m}^{1 / 2}\right)$ for the following initial data:

TABLE I. SOLUTIONS CORRESPONDING TO THE INITIAL DATA, OBTAINED BY NUMERICAL INTEGRATION.

\begin{tabular}{|l|c|c|}
\hline \multicolumn{1}{|c|}{ Case } & $\boldsymbol{a}$ & $\boldsymbol{b}$ \\
\hline$\omega_{10}, s^{-1}$ & 5 & 70 \\
\hline$\omega_{30}, s^{-1}$ & 120 & 5 \\
\hline$\gamma_{30}$ & 0.02 & 0.05 \\
\hline
\end{tabular}

In all cases of $\omega_{20}=0, \gamma_{20}=0$, the value of $\gamma_{10}$ was determined by the geometrical integral, and the initial slip velocity was given by equality $v=0, \varepsilon_{0}=-0.7 \cdot 10^{-4} \mathrm{~m}$. 

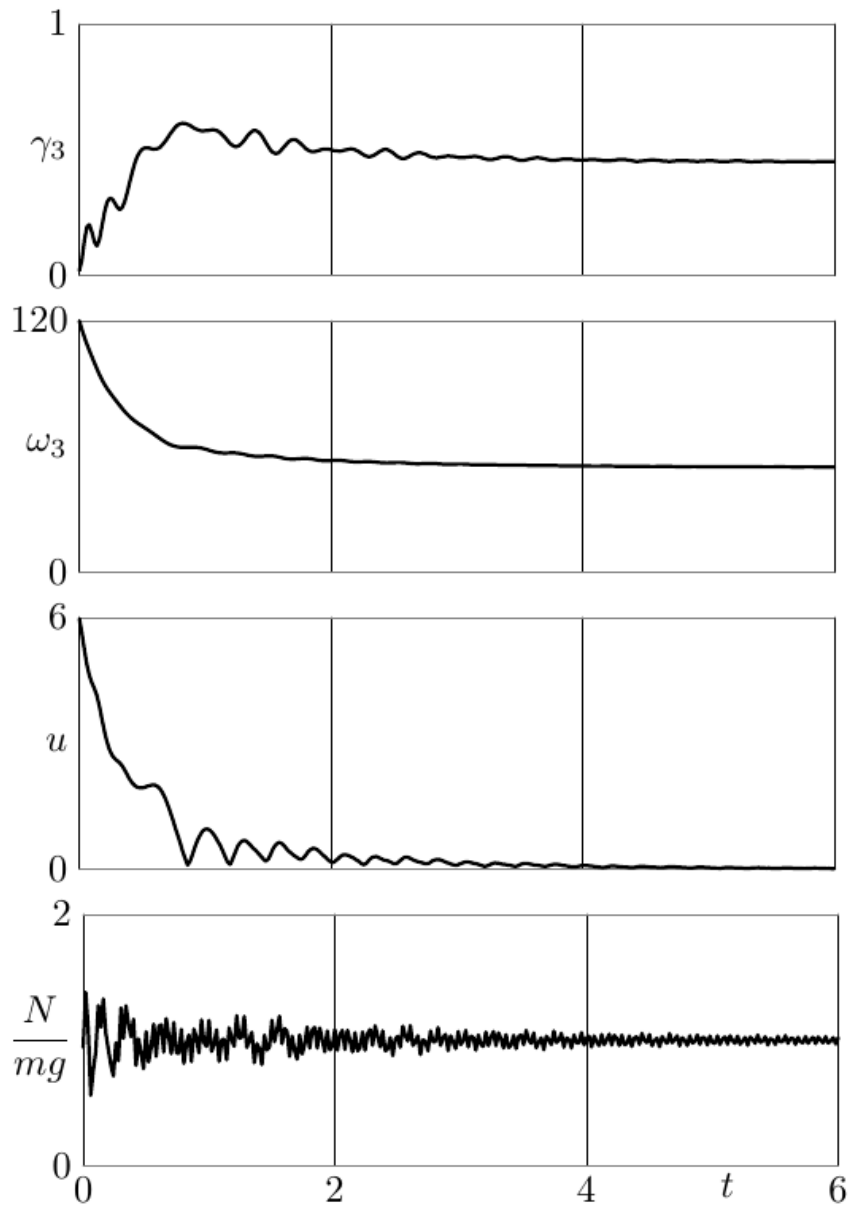

Fig. 3. Similar results corresponding to initial data b (table 1).

In Fig. 2, there are the time dependences of the cosine of the nutation angle of the ellipsoid, the projection of the angular velocity on the axis of symmetry, the slip velocity and the ratio of the support reaction to the gravity force of the ellipsoid, in the solutions corresponding to the initial data, (table 1) obtained by numerical integration. The motion begins in the neighbourhood of solution (8) (unstable rotation) but reaches stable rotation (7) during the evolution process.

In Fig. 3 there are similar results corresponding to initial data $b$ (table 1). The motion begins in the neighbourhood of solution (9) (unstable steady motion), but reaches stable presession (10) during the evolution process.

Let us note that in the case of displaced mass centre, it is possible to represent a geometrical interpretation of the ellipoids dynamic using generalized Smale diagrams [10].

\section{Acknowledgment}

This research was financed by the Russian Foundation for Basic Research (16-01-00338).

\section{References}

[1] M.A. Munitsyna, "The motions of a spheroid on a horizontal plane with viscous friction", Journal of applied mathematics and mechanics, vol. 76(2), pp. 154-161, 2012.

[2] A.V. Karapetyan, "The realization of non-holonomic constraints by viscous friction forces and the stability of celtic stones", Journal of applied mathematics and mechanics, vol. 45(1), pp. 42-51, 1981.

[3] A.V. Karapetyan, "The stability of the steady motion of systems with friction", Journal of applied mathematics and mechanics, vol. 51(4), pp. 552-558, 1987.

[4] A.V. Karapetyan, "The regular precession of a body on a horizontal plane with friction",Journal of applied mathematics and mechanics, vol 46(4), pp. 568-572, 1982

[5] E. I. Jury. Inners and stability of dynamic systems, Wiley, london, 1974.

[6] A.V. Karapetyan, A.A. Zobova, "The construction of poincar-chetayev and smale bifurcation diagrams for conservative non-holonomic systems with symmetry", Journal of applied mathematics and mechanics vol.69(2), pp. 202-214, 2005.

[7] A.V. Karapetyan, "On permanent rotations of a heavy solid on an absolutely rough horizontal plane", Journal of applied mathematics and mechanics, vol. 45(5), pp. 808-814, 1981.

[8] H. Hertz Die prinzipien der mechanik in neuem zusammenhange dargestellt. 1984.

[9] G. Wanner, E. Hairer Solving ordinary differential equations ii: Stiff and differential-algebraic problems. 2nd ed. Berlin, New york: Springerverlag, 1996.

[10] M.A. Munitsyna, A.V. Karapetyan, "The dynamics of a non-uniform spheroid on a horizontal plane", Journal of applied mathematics and mechanics, vol. 78(3), p. 228-232, 2014. 